\title{
Las variables macroeconómicas y la demanda de la industria hotelera mexicana, periodo 2010-2016
}

\section{Macroeconomic variables and demand in the Mexican hotel industry, period 2010-2016}

Oscar Naum Velázquez López ${ }^{1}$

Hotel Barceló Gran Faro Los Cabos

oscarnaum@barcelo.com

María Luisa Saavedra García 2*

Universidad Nacional Autónoma de México

Isaavedra@fca.unam.mx

Miriam Edith Saavedra García ${ }^{3}$

Universidad del Mar

medith@huatulco.umar.mx
* Autor corresponsal.

1 Revenue Manager, Paseo de las Misiones \# 926 Int 1-2, Campo de Golf. San José del Cabo, C.P. 23400, BCS, MÉXICO.

2 Facultad de Contaduría y Administración, Circuito Exterior s/n, Ciudad Universitaria, C.P. 04510, Ciudad de México, MÉXICO.

3 Instituto de Turismo, Ciudad Universitaria, Santa María Huatulco, C.P. 70989, Oaxaca MÉXICO.

\section{Resumen}

Propósito: El objetivo de esta investigación consistió en determinar el impacto de la variación de las principales variables macroeconómicas en la demanda del sector hotelero mexicano.

Metodología: Se analizaron datos de empresas cotizadas en la Bolsa Mexicana de Valores, por los años 2010-2016. Se utilizó un análisis de regresión múltiple, considerando como variable dependiente los cuartos ocupados y como variables independientes el tipo de cambio, el precio del petróleo y la inflación anual.

Resultados: Los principales hallazgos muestran que la variación en el número de Cuartos-noche ocupados es explicada por el tipo de cambio en $67 \%$, mientras que el precio del petróleo explica el $45.8 \%$.

Implicaciones: Los resultados implican la incidencia del tipo de cambio en el comportamiento de los ingresos y la importancia del precio del petróleo para el sector.

Originalidad/Valor: Este estudio marca la pauta para realizar un análisis completo del sector considerando los impactos macroeconómicos.
INFORMACIÓN ARTÍCULO

Recibido: 21 de Octubre 2021 Aceptado: 20 de Diciembre 2021

Palabras Claves:

Turismo

Entorno macroeconómico

Hoteles

Tipo de cambio

Petróleo 


\begin{abstract}
Purpose: The objective of this research was to determine the impact of the variation of the main macroeconomic variables on the demand of the Mexican hotel sector.

Methodology: Data from companies listed on the Mexican Stock Exchange were analyzed for the years 2010-2016; A multiple regression analysis was used, considering occupied rooms as the dependent variable and the exchange rate, oil price and annual inflation as independent variables.

Results: The main findings show that the variation in the number of occupied rooms-nights is explained by the exchange rate at $67 \%$, while the price of oil explains $45.8 \%$.

Implications: The results imply the impact of the exchange rate on the behavior of the income of this sector, and also show the importance of the price of oil for the sector.

Originality / Value: This study sets the standard for a complete analysis of the sector considering the macroeconomic impacts.
\end{abstract}

\section{ARTICLE INFO}

Received: 21 October 2021

Accepted: 20 December 2021

Keywords:

Tourism

Macroeconomic environment

Hotels

Exchange rate

Petroleum

\section{INTRODUCCIÓN}

El precio de los servicios turísticos se eleva por la depreciación del tipo de cambio, debido a que se encuentra vinculado a precios internacionales. Por lo que al final de la cadena de consumo del servicio, el turista local es quien asume el sobrecosto de la depreciación de la moneda del país que visita. El turismo nacional registra el $80 \%$ del consumo y el valor de los servicios del sector turístico y representa cerca del $88 \%$ del Producto Interno Bruto (PIB) de toda la actividad turística del país, según el Fondo Nacional de Fomento al Turismo (Fonatur, 2020).

En tanto, cuando se aprecia el tipo de cambio los turistas extranjeros no se sienten incentivados a visitar y gastar en México, puesto que sus dólares valen menos (García, Carranco y Martínez, 2009). Por otra parte, el turista nacional tiene un motivo más para salir de México debido a la apreciación del tipo cambiario que le beneficia, pero que desajusta la balanza turística.

Así pues, García y cols. (2009) refieren que si no se crea una estrategia para incentivar que el mercado nacional consuma los productos turísticos que se encuentran dentro del territorio local, las fluctuaciones cambiarias del peso mexicano frente al dólar estadounidense afectan el desempeño de este sector en las cuentas nacionales. De la misma manera, si no se tienen estrategias sólidas para aprovechar las fluctuaciones cambiarias encaminadas a crear un mercado interno sólido en la industria hotelera, se tiene una debilitación de esta y un panorama adverso para los consumidores nacionales.

Esta investigación tiene como objetivo determinar el impacto que se tiene en la demanda de la industria hotelera en México, frente a las fluctuaciones en las variables macroeconómicas, así como la relación existente entre el tipo de cambio y distintos indicadores financieros de empresas hoteleras que cotizan en la Bolsa Mexicana de Valores (BMV) y los indicadores de demanda del sector.

\section{MARCO TEÓRICO}

\section{La importancia del turismo en México}

La variedad y diversidad de recursos naturales y culturales con los que cuenta México, le ha permitido contar con una infraestructura turística creciente, pues que es uno de los sectores importantes para la recepción de inversión extranjera directa, posicionándose en el territorio nacional a través de cadenas hoteleras que se han establecido principalmente en destinos de playas tradicionales y nuevos destinos, con proyectos de resorts y proyectos afines a gran escala. A su vez, México cuenta con una ubicación estra- 
tégica en relación al mercado de Estados Unidos de Norteamérica, siendo los ciudadanos de este país una proporción importante de quienes lo visitan (alrededor de 50\%), los cuales tienen para elegir entre 70 destinos turísticos (Valverde y Benavides, 2017).

El sector turístico en México emplea casi cuatro millones y medio de personas, además de cumplir un papel fundamental de ser empleador de jóvenes y con una presencia importante de mano de obra femenina (De la Madrid, 2016; INEGI, 2019; Vázquez-Martínez, 2019). Es un sector que se considera de alta importancia puesto que genera $8.7 \%$ del PIB nacional, considerándose como impulsor del desarrollo regional, atrayendo inversiones capaces de producir cambios estructurales que permitan generar empleos y riqueza en las regiones donde se desarrolla, logrando un proceso permanente de progreso en la sociedad que habita ese ámbito (INEGI, 2019; Vázquez-Martínez, 2019; Medina, 2020).

Por lo anterior, la actividad de este sector "tiene una influencia importante sobre indicadores tanto cuantitativos (PIB, generación de empleo, entre otros), como cualitativos (calidad de vida, bienestar social, migración, etcétera), de los lugares geográficos donde opera" (Marquina, 2014, p.5). México recibió en el año 2019, 45 millones de turistas extranjeros, mostrando un incremento respecto del año 2018 en el que recibió 42 millones, lo que hace al turismo junto con las remesas extranjeras, los ingresos petroleros y las exportaciones del sector automotriz, un receptor importante de divisas extranjeras (INEGI, 2019).

Por su parte, Kido, Juárez, Domínguez y Sánchez (2016) mencionan que los impactos directos, indirectos e inducidos de este sector ante la ausencia de la actividad turística, serían los siguientes:

- El ingreso en el total de la economía disminuiría en un $13.68 \%$.

- Un debilitamiento del valor agregado de la producción nacional en $10.27 \%$.

- Reducción del empleo en general de un $14.6 \%$.

Del mismo modo, la actividad turística también tiene otros impactos negativos en los destinos receptores (Marquina, 2014), como pueden ser:

- El aumento general de precios, donde la población es la primera en resentirlo. Igualmen- te, al convertirse el suelo urbanizable en un bien escaso, su valor también aumenta.

- Los costos de oportunidad de esta actividad, puesto que al desarrollarla se puede dejar otras actividades al margen.

- Fuerte dependencia de la economía local, al ser la demanda turística muy volátil y estacional, haciéndola muy sensible la variación de precios y afectándole también los destinos y tendencias turísticas de moda.

Asimismo, los impactos positivos de la actividad turística mencionados por Marquina (2014) son:

- La inyección de ingresos para la economía de una región: A nivel nacional estos ingresos inicialmente mejoran la balanza de pagos y, posteriormente, estimulan la inversión propia del país y de los inversores extranjeros.

- La nueva actividad turística puede representar en un primer momento un estímulo a la agricultura y/o la industria, lo que permite diversificar las fuentes de ingreso tradicionales. La estacionalidad de las demandas turísticas y agrícolas contribuye a mantener una fuente constante de ingresos. Además no hay que olvidar que es más fácil ejercer un mayor control sobre los precios turísticos, y por ende, sobre los ingresos, que sobre los precios de las actividades tradicionales.

- La creación de empleo, tanto en el sector turístico como en el no turístico: En las zonas receptoras se pueden producir fuertes incrementos en los niveles de empleo. Este hecho se debe básicamente a que la actividad turística es una actividad esencialmente de servicios intensivos en manos de obra. No obstante, primeramente se tendió a la creación de espacios de trabajo con escasa o nula calificación, la directriz actual es la contraria y se trata de que la formación y especialización de recursos humanos sea la apropiada. Sin embargo, dicho empleo tiene el gran problema de que suele ser de un elevado grado de estacionalidad. El empleo generado por la actividad turística, puede ser encasillado, como el gasto, en tres tipos:

a) Directo: resultado de los gastos de los visitantes en instalaciones turísticas.

b) Indirecto: turístico pero no como resultado de lo anterior. 
c) Inducido: como resultado del gasto de los residentes debido a los ingresos por turismo.

- La creación y/o mejora de la infraestructura que requiere para su desarrollo, de las que se benefician tanto los visitantes como el resto de sectores económicos y los residentes locales.

- El poder de mejora de distribución de la renta, tanto a nivel de la población como a nivel espacial o regional (p. 14).

En este sentido:

en México el crecimiento económico puede impulsarse desarrollando el turismo interno, debido a que éste se concentra en temporadas ya identificadas en el año, por ejemplo, durante los meses de enero, mayo, junio, septiembre y octubre, se observa una baja ocupación hotelera, donde los operadores pueden ofertar destinos a los turistas nacionales (De la Madrid, 2016, p.54).

El Producto Interno Bruto Turístico (PIBT) de acuerdo con el Instituto Nacional de Estadística Geografía e Informática (INEGI) (2019), para el año 2018 tiene como primer componente los servicios de alojamiento con $28.1 \%$, seguido de servicios de transporte que alcanzan el $18.8 \%$, el servicio de restaurantes, bares y centros nocturnos aportan $15.2 \%$, mientras que los bienes y artesanías alcanzan el $11.7 \%$, y el comercio aporta un $8.1 \%$. Con esta conformación se muestra lo importante que resulta estudiar los servicios de alojamiento.

Cuando la moneda nacional se deprecia, se exportan bienes con precios más bajos y consecuentemente aumentan las ventas de las empresas que tienen negocios con el extranjero, de igual manera, aquellas empresas que poseen activos en otras divisas como las cuentas por cobrar en moneda extranjera, tendrán ganancias cambiarias al darse una depreciación de la moneda local, dado que al convertir las divisas estas tendrán mayor valor en moneda local. Por el contrario, al tener una apreciación de la moneda local tendrán pérdidas cambiarias (Torres, 2007; Juárez, 2007; Nava, 2012; Morales, Velásquez y García, 2016).

De acuerdo con Vargas (2010) y Mántey (2013), la devaluación de la moneda únicamente generará un incremento en las exportaciones si se cumplen las siguientes condiciones:

- Los precios de los bienes a exportar están expresados en moneda local
- Existe elasticidad al precio con respecto la demanda del exterior

- Existe elasticidad de la oferta de bienes a exportarse

Se puede agregar una cuarta condición para que se cumpla el incremento de las exportaciones de acuerdo con Berman y Berthou (2009), la depreciación no se debe transmitir a los precios domésticos.

\section{Factores macroeconómicos que afectan la de- manda turistica}

Al sector turístico le afectan factores en los que se incluyen problemas geopolíticos, políticas macroeconómicas, relaciones bilaterales con otros países y políticas comerciales y de visado (Muhammand, Mohammad, Ghulam, Mubbshar y Salman, 2018). Es así como Witt y Martin (1987) exponen que los gastos en turismo involucran dos elementos clave que son los costos de hospedaje y los costos del transporte que prevalecen en el país que se va a visitar. De igual modo, un mayor nivel de inflación resulta en costos más altos de vida y de viaje, lo que disminuye el flujo de turistas extranjeros como locales, sea que se traten de viajes de negocios o placer.

En tanto, Tang y Lean (2007) sugieren que un incremento en la inflación trae como consecuencia un aumento en el costo de vida, que al final lleva a una disminución en el poder de compra y esto a su vez afecta negativamente el flujo de turistas. Entre estudios que se han llevado a cabo para identificar las causas que incrementan la demanda turística de un país en particular, se encuentran los de Quayson y Var (1982), quienes expusieron que estimar la demanda de turismo es el factor más importante para cualquier país que intente atraer turistas locales e internacionales, para asegurarse que los recursos disponibles son efectiva y eficientemente utilizados.

En este sentido, la literatura actual ha identificado varios factores que determinan la demanda turística: inflación, tipo de cambio, precios del petróleo, cambio climático, producción y utilización energética, estabilidad política, terrorismo, corrupción, ingreso, crecimiento económico e infraestructura para facilitar al turista su viaje (Jiménez, Peña y Ruiz, 2017; Muhammand y cols., 2018). Investigadores han discutido frecuentemente que el tipo de cambio puede ser el elemento que más determina la demanda turística, mientras que los estudios sugieren que se incluya 
el tipo de cambio en la ecuación de demanda turística para evitar el problema potencial de omisión de variables (Balaguer y Cantavella-Jordá, 2002).

Lo anterior, debido a que la devaluación de la moneda local resulta en una disminución general en los gastos de los extranjeros que tienen una moneda fuerte y están deseosos de visitar México. Como consecuencia, el flujo de turistas a México se incrementa rápidamente. Así lo corroboran Archibald y Lacorbinière (2008), quienes exponen que una depreciación en el tipo de cambio está positivamente asociada con la demanda turística, puesto que viajar hacia los países que tienen su moneda devaluada resulta menos costoso.

Estudios empíricos han mostrado que las fluctuaciones en el tipo de cambio están asociadas con variaciones en las llegadas internacionales de turistas, una devaluación del tipo de cambio en el país de destino atrae flujos hacia este, mientras que una devaluación en el país de origen reduce las salidas de turistas (Agiomirgianakis, Serenis y Tsounis, 2014; Agiomirgianakis, Serenis y Tsounis, 2015), lo cual implica que un incremento en el ingreso del país de origen afecta positivamente la posibilidad de su población para viajar al extranjero. El tipo de cambio puede también ser usado frecuentemente como una variable separada en el modelo de demanda del turismo, porque la gente puede mostrar distintos comportamientos a la variación real de los precios contra la volatilidad de la tasa de cambio (Muhammand y cols., 2018).

Para Muhammand y cols. (2018), el impacto más grande en la demanda turística viene de los tipos de cambio, porque un incremento en la tasa de cambio significa que la depreciación en la moneda del país anfitrión tiene una asociación positiva con la demanda turística. En contraparte, una apreciación en el tipo de cambio tiene una asociación negativa con la demanda turística. En tanto, una depreciación de la moneda de una nación hace que el turismo internacional que ingresa tenga precios más accesibles y por eso hace más atractivo para los viajeros visitar ese país, caso contrario, un incremento en el valor de la moneda de un país hará al turismo mundial más costoso y causará una disminución en los visitantes extranjeros (Muhammand y cols., 2018).

Por otra parte, Becken y Lennox (2012) exponen que el transporte es fundamental para mejorar el turismo, siendo que este depende en gran medida de la energía, y, principalmente del petróleo, lo cual implica que siendo los costos de transportación una parte integral de lo que implica viajar a un destino, su incremento es consecuencia directa del aumento de los precios del petróleo y se encuentra relacionado negativamente con los flujos de turistas (Agiomirgianakis y cols., 2015).

En consecuencia, el aumento que los precios del petróleo tienen en la demanda turística repercute en el mercado local puesto que un aumento en los precios reduce el consumo e inversión local, aparte de esto, los altos precios del petróleo reducen el poder adquisitivo de la moneda local y la posibilidad de los consumidores de comprar bienes y servicios (Anoruo y Elike, 2009). Yeoman, Lennon, Blake, Galt, Greenwood y McMahon-Beattie (2007) examinaron los nexos de los precios del petróleo y la demanda turística, concluyendo que, al incrementar los precios del petróleo se afectaba inversamente y disminuía la demanda turística.

La Organización Mundial del Turismo (OMT) en 2020, también reportó una influencia negativa de los precios del petróleo en el turismo, no obstante, las investigaciones sugieren diferentes efectos para distintos países. Por una parte, un incremento en el precio del petróleo ocasiona una tasa de inflación al alza, lo cual puede ir en detrimento de la demanda turística porque las actividades relacionadas al turismo generalmente son grandes consumidoras de petróleo (Muhammand y cols., 2018).

Como un daño directo se encuentra el incremento de la inflación como resultado de un aumento en los precios del petróleo, sin embargo, este puede tener un efecto positivo en los países productores de petróleo, dado que mientras más alto sea el precio de este, más alto será el ingreso para los países (Becken y Lennox, 2012). Sin embargo, los resultados de las investigaciones empíricas de Muhammand y cols. (2018), dejan en evidencia que un incremento o decremento en los precios del petróleo tienen un efecto negativo en el turismo, esto debido a que el precio del petróleo puede impactar las operaciones financieras y turísticas por el impacto que estos trasladan al transporte, costos de generación, vulnerabilidad monetaria y los flujos de efectivo ilimitados.

Por último, en economías en desarrollo, la inflación es el mayor determinante de la demanda turística, las tendencias inflacionarias internas, $y$, sobre todo las externas afectan al turismo internacional. Un estudio de Muhammand y cols. (2018), encontró que la disminución en la inflación 
tiene un efecto negativo en la demanda turística en el largo plazo, mientras que un incremento en la inflación también tiene un impacto negativo en el turismo. Sin embargo, la disminución en inflación tiene mayor influencia en la demanda turística en comparación con un aumento en la inflación, esto se debería a que la inflación está directamente asociada con el poder de compra de los consumidores, puesto que cuando la inflación incrementa, el poder de compra de los turistas baja y estos no visitan los destinos donde encuentran costoso vivir y viajar. Caso contrario, cuando la inflación disminuye más turistas visitan los países donde encuentran los costos de transportación y de vida más baratos.

Entonces, para determinar la relación entre la demanda turística y las principales variables macroeconómicas se tomará como variable dependiente el dato de los cuartos-noche ocupados y como variables independientes el tipo de cambio, el precio del petróleo y la inflación anual. A continuación, se presenta el modelo teórico (ver Figura 1).

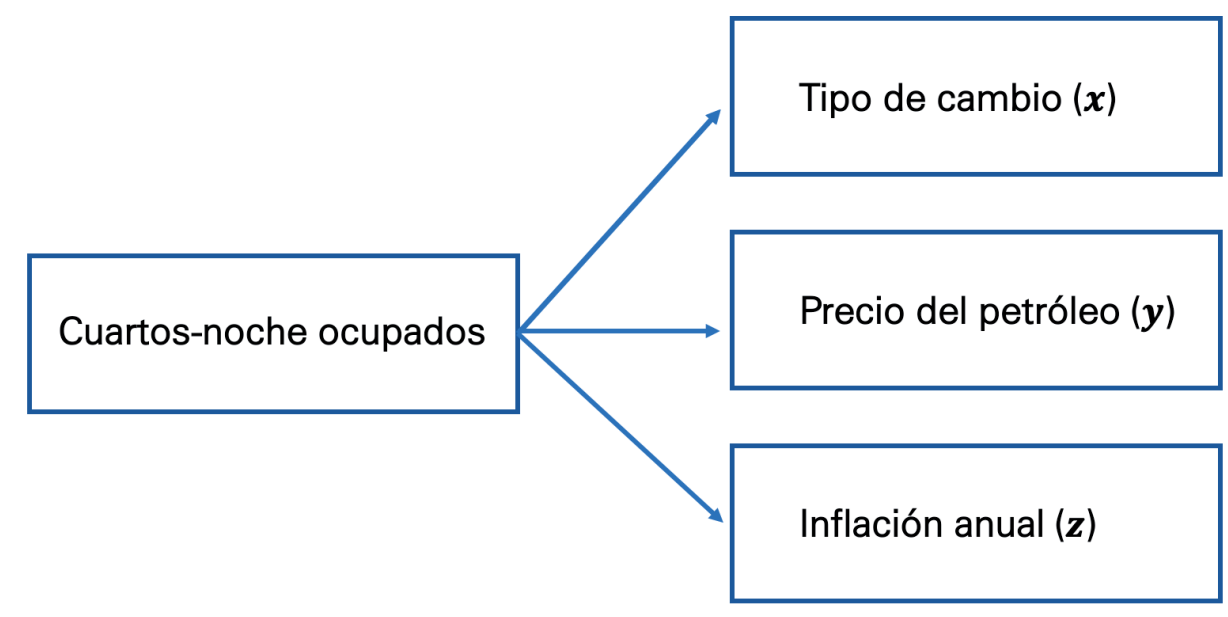

Figura 1. Modelo teórico. Fuente: elaboración propia.

\section{MÉTODO}

Se realizó un estudio correlacional causal para conocer el impacto que tiene la fluctuación del tipo de cambio del peso frente al dólar estadounidense en la industria turística, se tomaron en cuenta distintos indicadores de mercado dentro de dicha industria (SECTUR, 2014, 2016, 2019). De igual manera, se describe el impacto que tres indicadores macroeconómicos tienen en el comportamiento del mercado nacional en esta industria, y si se puede predecir dicho comportamiento de la demanda en un tiempo determinado. Los datos de estos indicadores fueron tomados desde el Banco de México (2018a, 2018b) y del Sistema Integral sobre Economía Minera (SINEM) (2019), los precios históricos diarios petróleo West Texas Intermediate (WTI), Brent y Mezcla Mexicana de Petróleo.
Se recabaron datos en valores nominales de enero 2010 a diciembre 2016, para evaluar el comportamiento de los distintos indicadores macroeconómicos de manera mensual (tipo de cambio peso mexicano/dólar americano, inflación anual y precio del barril de petróleo), de mercado de manera mensual (volumen de visitantes, cuartos ocupados, turistas-noche y gasto promedio) y financieros de manera trimestral (ingresos, Earnings before interest, taxes, depreciation and amortization (EBITDA por sus siglas en inglés), costo de ventas y otros gastos operativos), tanto de las bases de datos y boletines de INEGI, Banco de México y SECTUR, así como estados financieros de las empresas hoteleras listadas en la BMV (Tabla 1). 
Tabla 1. Operacionalización de variables.

\begin{tabular}{lll}
\hline \multicolumn{1}{c}{ Variables } & \multicolumn{1}{c}{ Dimensiones } & \multicolumn{1}{c}{ Indicadores } \\
\hline & Volumen de visitantes nacionales & Número de personas \\
Comportamiento del mercado & Cuartos ocupados por turistas nacionales & Número de cuartos \\
& Turistas nacionales por noche & Número de personas \\
& Gasto & Valor en pesos mexicanos (miles) \\
\hline Variables financieras del sector & $\begin{array}{l}\text { Ingresos (I) } \\
\text { EBITDA (utilidades antes de intereses, im- }\end{array}$ & Pesos mexicanos (millones) \\
& Costo de ventas (CV) & Pesos mexicanos (millones) \\
& Otros gastos operativos (GO) & Pesos mexicanos (millones) \\
& Tipo de cambio peso dólar (TC) & Pesos mexicanos (millones) \\
\hline Variables macroeconómicas & Precio del petróleo (P) & Pesos mexicanos \\
& Inflación anual (IA) & Precio del barril de petróleo, en US \\
dólares americanos.
\end{tabular}

Fuente: elaboración propia.

Para las variables financieras se tomaron datos del $100 \%$ de las empresas públicas del sector hotelero que cotizan en la BMV:

- GRUPE, S.A.B. DE C.V.

- HOTELES CITY EXPRESS, S.A.B. DE C.V.

- GRUPO hotelero SANTA FE, S.A.B. DE C.V.

- grupo posadas, S.A.B. DE c.V.

- RLh PROPERTIES, S.A.B. DE C.V.

\section{RESULTADOS}

Se ha observado que el comportamiento de la industria turística se ve afectado por las temporadas vacacionales y por picos que se presentan a lo largo del año, por lo que, tanto para las variables de mercado como para las variables financieras se procedió a desestacionalizar las series de datos, antes de aplicar el tratamiento estadístico.

\section{Análisis descriptivo}

Con el fin de comprender la naturaleza de las variables que se utilizaron en el estudio y las observaciones realizadas, se presenta a continuación un resumen de la estadística descriptiva de cada una de estas.

En la Tabla 2 se observa que el volumen de visitantes es en promedio 6.5 millones al mes, mientras que los cuartos ocupados ascienden a 5.7 millones mensuales. Los turistas por noche ascienden a 11 millones, y el gasto promedio asciende a 17,204 dólares americanos. La varianza y la desviación estándar muestran alta dispersión de los datos, lo que implica el comportamiento heterogéneo de estos indicadores. Se trabajó con 84 observaciones, dado que se contabilizaron los datos de manera mensual por siete años. 
Tabla 2. Análisis descriptivo del comportamiento del mercado.

\begin{tabular}{lrrrr}
\hline \multirow{2}{*}{ Descriptivos } & \multicolumn{4}{c}{ Comportamiento del mercado } \\
\cline { 2 - 5 } & $\begin{array}{c}\text { Volumen de } \\
\text { visitantes }\end{array}$ & Cuartos ocupados & Turistas por noche & $\begin{array}{c}\text { Gasto } \\
\text { U\$ }\end{array}$ \\
\hline Media & 6540702 & 5754562 & 11040016 & 17,204 \\
Varianza & $4.14 \mathrm{E}+11$ & $3.86 \mathrm{E}+11$ & $1.33 \mathrm{E}+12$ & 26,408 \\
DS & 643744 & 621304 & 1153340 & 1.35 \\
No & 84 & 84 & 84 & 84 \\
\hline
\end{tabular}

Nota: DS: Desviación estándar; No: Número de observaciones. Para el gasto se utilizó el tipo de cambio promedio anual, para el período bajo estudio (2010-2016).

Fuente: elaboración propia según los resultados de cálculos estadísticos.

En la Tabla 3 se muestra el análisis descriptivo de las variables financieras, las cuales se consideraron con el fin de tratar de determinar si existe relación entre estas y las variables macroeconómicas, así como las variables del comportamiento del mercado. Se puede observar que los ingresos promedio trimestrales ascienden a 41.2 millones de dólares americanos, mientras que el EBITDA es de 8.7 millones de dólares americanos, en tanto que el costo de ventas reporta 29.3 millones de dólares americanos y los gastos operativos 5.8 millones de dólares americanos al trimestre. La varianza y la desviación estándar dan cuenta de la alta dispersión de los datos al mostrar el sector un comportamiento estacional. Las observaciones son 28, las cuales corresponden a los trimes- tres que se encuentran comprendidos en los siete años del estudio, dado que las empresas reportan sus datos de modo trimestral.

En cuanto a las variables macroeconómicas se observa que el tipo de cambio promedio mensual en el período de estudio fue de 14.12 pesos mexicanos por dólar estadounidense, mientras que el precio del petróleo promedio fue de 77.06 dólares americanos por barril, y la inflación anual 3.58\% promedio mensual, mostrándose también una alta dispersión de los datos por la fluctuación de estos indicadores, habiéndose utilizado 84 observaciones mensuales que corresponden al periodo de estudio.

Tabla 3. Análisis descriptivo de las variables financieras del sector y las variables macroeconómicas.

\begin{tabular}{|c|c|c|c|c|c|c|c|}
\hline \multirow{2}{*}{ Descriptivos } & \multicolumn{4}{|c|}{$\begin{array}{l}\text { Variables financieras del sector } \\
\text { (Millones de U\$) }\end{array}$} & \multicolumn{3}{|c|}{ Variables macroeconómicas } \\
\hline & Ingresos & EBITDA & $\begin{array}{l}\text { Costo de } \\
\text { ventas }\end{array}$ & $\begin{array}{l}\text { Otros gastos } \\
\text { operativos }\end{array}$ & $\begin{array}{l}\text { Tipo de } \\
\text { cambio }\end{array}$ & $\begin{array}{l}\text { Precio del } \\
\text { petróleo }\end{array}$ & $\begin{array}{l}\text { Inflación } \\
\text { anual }\end{array}$ \\
\hline Media & 41.20 & 8.70 & 29.36 & 5.80 & 14.12 & 77.06 & 3.58 \\
\hline Varianza & $5,939.82$ & 136.04 & $1,571.50$ & 275.83 & 5.00 & 726.77 & 0.45 \\
\hline DS & 20.51 & 3.10 & 10.55 & 6.35 & 2.23 & 26.96 & 0.67 \\
\hline No & 28 & 28 & 28 & 28 & 84 & 84 & 84 \\
\hline
\end{tabular}

Nota: DS: Desviación estándar; No: Número de observaciones. Para las variables financieras, se utilizó el tipo de cambio promedio anual, para el período bajo estudio (2010-2016).

Fuente: elaboración propia según los resultados de cálculos estadísticos.

\section{Análisis correlacional}

Los resultados de la correlación del comportamiento de las variables del mercado turístico y el tipo de cambio se presentan en la Tabla 4, la cual muestra los coeficientes de relación entre las variables consideradas para el análisis del com104 portamiento del mercado. El resultado muestra una relación positiva entre la variación del tipo de cambio y el comportamiento del mercado turístico, coincidiendo con Vargas (2010) en referencia a que, al darse una devaluación del peso mexica- 
no, el consumo del mercado internacional se incrementa al abaratarse los servicios, algo similar sucede con el turismo nacional al resultar más económico consumir en el mercado local que en el externo.

Como consecuencia, el volumen de visitantes muestra igualmente una relación positiva de 0.8152 , al igual que los cuartos ocupados con una relación de 0.8280 y turistas noche similar a las anteriores, muestra una relación positiva con el tipo de cambio con 0.7391.Finalmente, el gasto promedio tiene una relación positiva de 0.8065 con respecto al tipo de cambio, siendo estos hallazgos coincidentes con lo expresado por Balaguer y Cantavella-Jordá (2002) y Archibald y Lacorbinière (2008), Agiomirgianakis y cols. (2014) y Muhammand y cols. (2018), sobre el importante impacto del tipo de cambio en las variables de la demanda turística.

Tabla 4. Relación del comportamiento del mercado y tipo de cambio.

\begin{tabular}{|c|c|c|c|c|c|}
\hline Ítems & Pesos por dólar & Volumen de visitantes & Cuartos ocupados & Turistas noche & Gasto \\
\hline Pesos por dólar & 1.0000 & & & & \\
\hline Volumen de visitantes & 0.8152 & 1.0000 & & & \\
\hline Cuartos ocupados & 0.8280 & 0.9813 & 1.0000 & & \\
\hline Turistas noche & 0.7391 & 0.9595 & 0.9590 & 1.0000 & \\
\hline Gasto & 0.8065 & 0.6095 & 0.5650 & 0.5378 & 1.0000 \\
\hline
\end{tabular}

Fuente: elaboración propia según los resultados de cálculos estadísticos.

La relación entre las variables financieras de las empresas del sector y el tipo de cambio se muestra en la Tabla 5. En ella, se expresan los coeficientes de relación entre las variables consideradas para el análisis de los datos financieros de las empresas hoteleras listadas en la BMV. Existe una relación positiva media baja entre la variación de la fluctuación del peso mexicano y el comportamiento de los resultados financieros en los indicadores de EBITDA y otros gastos operativos. Esto implicaría que la fluctuación del tipo de cambio tiene un impacto medio en las utilidades y en los gastos operativos. Se encontró, además, una relación muy baja entre el tipo de cambio y los ingresos de las compañías hoteleras que cotizan en bolsa al ser esta 0.1027 , por lo que se concluye que los ingresos no necesariamente dependen de la fluctuación que haya entre el tipo de cambio del peso mexicano y el dólar americano.

El costo de ventas tiene una relación positiva baja con el tipo de cambio: 0.1388 . Se puede inferir que, al ser un servicio prestado en territorio nacional, los costos se adquieren principalmente en moneda local, por lo que la fluctuación cambiaria no tiene una influencia directa muy representativa en estos indicadores.

Tabla 5. Relación de variables financieras del sector y tipo de cambio.

\begin{tabular}{|c|c|c|c|c|c|}
\hline Ítems & Pesos por dólar & Ingresos & EBITDA & Costo de ventas & $\begin{array}{c}\text { Otros gastos } \\
\text { operativos }\end{array}$ \\
\hline Pesos por dólar & 1.0000 & & & & \\
\hline Ingresos & 0.1027 & 1.0000 & & & \\
\hline EBITDA & 0.4499 & 0.7135 & 1.0000 & & \\
\hline Costo de ventas & 0.1388 & 0.8192 & 0.7549 & 1.0000 & \\
\hline $\begin{array}{l}\text { Otros gastos } \\
\text { operativos }\end{array}$ & 0.4118 & 0.7257 & 0.4888 & 0.3635 & 1.0000 \\
\hline
\end{tabular}

Fuente: elaboración propia según los resultados de cálculos estadísticos. 
En la Tabla 6 se presenta la relación entre el comportamiento del mercado y el precio del petróleo, y como se puede observar la relación es media, lo que implica que no es el principal determinante en la demanda interna del turismo en México. Y aunque influye en los costos de transportación y esto se encuentra relacionado negativamente con los flujos de turistas (Agiomirgianakis y cols., 2015), en el mercado interno no tiene tanta injerencia.
Sin embargo, una relación negativa con el gasto implica que al elevarse el precio del petróleo, el gasto disminuye al incrementarse el precio de los servicios turísticos, tal como refieren Anoruo y Elike (2009) y Yeoman y cols. (2007). Aunque la relación que se presenta es débil, muestra indicio de la relación.

Tabla 6. Relación entre el comportamiento del mercado y el precio del petróleo.

\begin{tabular}{lccccc}
\hline Ítems & Precio petróleo & $\begin{array}{c}\text { Volumen de } \\
\text { visitantes }\end{array}$ & Cuartos ocupados & Turistas noche & Casto \\
\hline Precio petróleo & 1.0000 & & & \\
Volumen de visitantes & 0.5795 & 1.0000 & 1.0000 & \\
Cuartos Ocupados & 0.5982 & 0.9813 & 0.9590 & 1.0000 \\
Turistas Noche & 0.6075 & 0.9595 & 0.5650 & 0.5378 & 1.0000 \\
Gasto & -0.1166 & 0.6095 & & \\
\hline
\end{tabular}

Fuente: elaboración propia según los resultados de cálculos estadísticos.

En la Tabla 7 se muestra la relación de las variables financieras del sector y el precio del petróleo, la cual es media negativa con los ingresos y resulta consistente con lo mencionado en la literatura previa, donde un aumento del precio del petróleo genera inflación y, en consecuencia, aumenten los costos de viaje (Agiomirgianakis y cols., 2015; Muhammand y cols., 2018), el costo de los bienes y servicios (Anoruo y Elike, 2009), y disminuya la demanda turística (Yeoman y cols., 2007; OMT, 2020). Lo anterior, repercute directamente en el ingreso de las empresas del sector turístico. Las otras variables muestran relaciones negativas, aunque débiles. Asimismo, dan un indicio del impacto negativo del alza del precio del petróleo en las variables financieras del sector.

Tabla 7. Relación entre variables financieras del sector y precio del petróleo.

\begin{tabular}{lccccc}
\hline \multicolumn{1}{c}{ Ítems } & Precio petróleo & Ingresos & EBITDA & $\begin{array}{c}\text { Costo de } \\
\text { ventas }\end{array}$ & Otros gastos operativos \\
\hline Precio petróleo & 1.0000 & & & & \\
Ingresos & -0.5380 & 1.0000 & & & \\
EBITDA & -0.2185 & 0.7135 & 1.0000 & 1.0000 & 1.0000 \\
Costo de ventas & -0.3090 & 0.8192 & 0.7549 & 0.3635 & 0.4888 \\
Otros gastos operativos & -0.3270 & 0.7257 & & \\
\hline
\end{tabular}

Fuente: elaboración propia según los resultados de cálculos estadísticos.

\section{Análisis de regresión múltiple}

Para determinar qué variables macroeconómicas influyen en la demanda turística, se planteó el modelo de regresión múltiple. Se trabajó con datos mensuales de enero 2010 a diciembre 2016, con fin de establecer la dependencia del número de cuartos-noche ocupados (demanda turística), con el tipo de cambio, el precio del petróleo y la inflación anual.

Se formula la siguiente hipótesis:

Ho: Los cuartos ocupados en el sector hotelero de la Ciudad de México no dependen del tipo de cambio, precio del petróleo e inflación anual. 
Al considerar a los Cuartos-noche ocupados mensualmente, la variable dependiente y el tipo de cambio, el precio del petróleo y la inflación, se obtuvo como resultado un valor de $R=0.9430$ y una $R^{2}$ ajustada de 0.8851 . Estos resultados permiten rechazar la hipótesis nula e inferir que el comportamiento de las variables tipo de cambio, precio del petróleo e inflación anual, explican en un 88\% la variación en los cuartos ocupados, comprobándose así el impacto del contexto macroeconómico en el comportamiento del sector hotelero en México. Por otra parte, el valor de $F$ es de 214.06 mientras el valor crítico de $F$ es de 4.07E-38, con lo que se confirma el rechazo de la hipótesis nula.

Con el fin de conocer el poder explicativo de cada variable independiente sobre la dependiente, en la Tabla 8 se muestran las betas de cada variable, donde se aprecia que la beta más elevada corresponde al tipo de cambio con 0.670 , mientras que el precio del petróleo muestra una beta de 0.458 , en tanto que la inflación anual muestra una beta negativa de -0.105. De acuerdo con las betas obtenidas de la regresión, el tipo de cambio explica en un $67 \%$ el número de Cuartos-noche ocupados mensualmente. Esta es la variable independien- te qué más influencia tiene sobre el comportamiento de nuestra variable dependiente, siendo coincidente estos resultados con los hallazgos de Balaguer y Cantavella-Jordá (2002) y Archibald y Lacorbinière (2008), Agiomirgianakis y cols. (2014) y Muhammand y cols. (2018), sobre que el cambio es la variable macroeconómica de mayor influencia en la demanda turística.

El Precio del petróleo explica en un $45.8 \%$ el número de cuartos noches ocupados, coincidiendo en estos resultados con los hallazgos de Anoruo y Elike (2009); Yeoman, y cols. (2007); Muhammand, y cols. (2018) y OMT (2020), con respecto al importante impacto que tienen los precios del petróleo en la demanda turística. Mientras que la inflación anual explica en un sentido negativo un $10.5 \%$, confirmando lo encontrado por Muhammand, y cols. (2018), puesto que los años analizados se caracterizaron por una disminución de la inflación que tuvo un efecto negativo en la demanda turística, porque una baja inflación representa menos dinamismo en la economía, considerando que predomina el turismo interno o nacional (ver Tabla 8).

Tabla 8. Coeficientes de la regresión múltiple.

\begin{tabular}{|c|c|c|c|c|c|}
\hline \multirow[t]{2}{*}{ Modelo } & \multicolumn{2}{|c|}{ Coeficientes no estandarizados } & \multirow{2}{*}{$\begin{array}{c}\text { Coeficientes } \\
\text { tipificados } \\
\text { Beta }\end{array}$} & \multirow[t]{2}{*}{$t$} & \multirow{2}{*}{ Sig. } \\
\hline & $\mathrm{B}$ & Error típico & & & \\
\hline (Constante) & 2659177.99 & 321830.22 & & 8.263 & 0.000 \\
\hline Tipo de cambio $(x)$ & 186149.44 & 13795.39 & 0.670 & 13.494 & 0.000 \\
\hline Precio del petróleo (y) & 10551.33 & 876.20 & 0.458 & 12.042 & 0.000 \\
\hline Inflación anual (z) & -96678.36 & 45184.21 & -0.105 & -2.140 & 0.035 \\
\hline
\end{tabular}

Fuente: elaboración propia según los resultados de cálculos estadísticos.

La ecuación de regresión es la siguiente:

Cuartos-noche ocupados $=2659177.99+1861149.44 x+10551.33 y-96678.36 z$

\section{DISCUSIÓN}

Para México, el turismo internacional es de gran importancia y representa una gran captación de divisas, sin embargo, el turismo nacional es un pilar tanto del sector como de la economía mexicana, puesto que el mercado local representa el $80 \%$ del total del consumo en la industria turística (INEGI, 2019; Vázquez-Martínez, 2019; Medina, 2020).
La devaluación de la moneda nacional hace que los productos exportables y exportados se vuelven más baratos para los compradores extranjeros, mientras que los productos importados, por el contrario, se encarecen para los consumidores nacionales. Estas condiciones fortalecen la capacidad competitiva para los productos y servicios 
locales ofrecidos por las empresas nacionales. Por el contrario, una apreciación de la moneda local provoca que los precios de los bienes y servicios locales cotizados en moneda extranjera se eleven, lo que produce disminución de las exportaciones y una contracción del mercado doméstico debido a que a los consumidores locales les resulta más barato comprar bienes y servicios del exterior (Torres, 2007; Juárez, 2007; Nava, 2012; Morales y cols., 2016).

Por lo tanto, la devaluación de la moneda local amplía los mercados externo e interno y contribuye a ampliar nuevos mercados en el corto plazo. Esto implica que el tipo de cambio puede ser el elemento que más determina la demanda turística debido a que una devaluación en la moneda local resulta en una disminución general en los gastos de los extranjeros que tienen una moneda más fuerte, lo que incrementa el flujo de turistas internacionales. Por otra parte, en lo que concierne al mercado local, al devaluarse la moneda se reduce la salida de turistas al extranjero y se incrementa el consumo de los servicios nacionales (Jiménez y cols., 2017; Muhammand y cols., 2018). Por consiguiente, resulta necesario que los empresarios del ramo se mantengan atentos a estas fluctuaciones, con el fin de tomar decisiones anticipadas que le permitan sobrevivir a una época de movimiento en el comportamiento del tipo de cambio.

\section{CONCLUSIONES}

La principal conclusión es que la demanda turística nacional es directamente proporcional a la depreciación del peso mexicano frente al dólar, esto debido a que a pesar de que la industria hotelera se rige por una determinación de precios dolarizada, el hecho de que el peso mexicano se haya debilitado frente al dólar norteamericano en los años 2010-2016, no tiene una repercusión negativa frente al comportamiento del mercado local. Puesto que, al haber un encarecimiento de los productos de turismo externo, el consumidor local se inclinará por los servicios ofrecidos en el país.

Asimismo, la fluctuación del tipo de cambio no afecta de manera negativa el comportamiento del mercado nacional, por el contrario, las relaciones que se encontraron entre el tipo de cambio y las variables que se definieron de mercado: son positivas y altas, con lo que se concluye que el mercado interno se fortalece al haber fluctuaciones al alza del dólar frente al peso. Es decir, mientras aumenta el tipo de cambio, el consumo del mercado local se acrecienta.

Por otra parte, se concluye que la depreciación del peso mexicano frente al dólar estadounidense no tiene una relación significativa con los resultados financieros de las empresas hoteleras listadas en la BMV, dado que las empresas realmente no se ven afectadas de manera negativa en sus resultados financieros por las fluctuaciones de tipo de cambio, puesto que, como señalan Guzmán, Leyva y Cárdenas (2007) si una empresa tiene obligaciones en moneda extranjera se incrementan sus riesgos financieros y además si utilizan insumos importados aumentan sus costos, lo cual a su vez decrementa sus utilidades. En este sentido, la prestación de servicios de hospedaje no incurre en gastos en moneda extranjera para su operación diaria, con la excepción de gastos en inversiones de capital que son extraordinarias y con largos periodos entre unas y otras.

Finalmente, en el análisis de las relaciones de las variables macroeconómicas con la demanda turística, se concluye que la variable macroeconómica que más efectos tiene en el comportamiento de la demanda del mercado nacional es el tipo de cambio, por lo que su análisis es de gran importancia para poder tomar decisiones financieras en las empresas hoteleras que operan en el país.

Los resultados de esta investigación abren oportunidades para ser aprovechadas por los prestadores de servicios hoteleros y por el Estado, con el fin de desarrollar más la industria y fortalecerla, al tener más consumidores locales y atractivos para visitantes extranjeros que obtienen un mayor beneficio al traer su divisa fortalecida al país. de esta forma, la industria puede obtener beneficios que, bien invertidos, pueden encaminar a un fortalecimiento de la economía local.

En tanto, los prestadores de servicios de hospedaje podrían pronosticar los flujos de turistas a los establecimientos de este tipo, para así tener estrategias financieras y de mercado, aprovechar dichos flujos, incentivar nuevos y aprovechar la derrama económica. Por otro lado, el gobierno puede conocer la demanda estimada de turistas y crear las condiciones necesarias en los destinos turísticos para soportar la afluencia de visitantes. 


\section{REFERENCIAS}

Agiomirgianakis, G., Serenis, D. y Tsounis, N. (2014). Exchange rate volatility and tourist flows into Turkey. Journal of Economic Integration, 2(4), 700-725.

Agiomirgianakis, G., Serenis, D. y Tsounis, N. (2015). The effects of exchange rate volatility on tourist flows: Evidence from the UK and Sweden. International Journal of Tourism Policy, 6(1), 1-16.

Anoruo, E. y Elike, E. (2009). An empirical investigation into the impact of high oil prices on economic growth of oil-importing African countries. International Journal of Economic Perspectives, 3(2), 121-129.

Archibald, X. y Lacorbinière, J. (2008, 28-31 of july). Analysis of Tourism competitiveness in the Caribbean: a Gravity Model approach. 29th Annual Review Seminar, Research Department, Central Bank of Barbados. Recovered from: http://www. centralbank.org.bb/news/article/6650/analysis-of-tourism-competitiveness-in-the-caribbean-a-gravity-model-approach

Balaguer, J. y Cantavella-Jordá, M. (2002). Tourism as a long-run economic growth factor: the Spanish case. Applied Economics, 34(7), 877-884.

Banco de México. (2018a). Inflación. Recuperado de: http://www.anterior.banxico.org.mx/portal-inflacion/inflacion.html

Banco de México (2018b). Mercado cambiario (Tipo de cambio). Recuperado de: http://www.anterior.banxico.org.mx/portal-mercado-cambiario/ index.html

Becken, S. y Lennox, J. (2012). Implications of a longterm increase in oil prices for tourism. Tourism Management, 33(1), 133-142.

Berman, N. y Berthou, A. (2009). Financial market imperfections and the impact of exchange rate movements. Review of International Economics, 17(1), 103-120.

De la Madrid, E. (2016). Turismo, la opción más visible para crecer y generar desarrollo en México. Comercio Exterior Bancomext, (abril-junio), 5055.

Fondo Nacional de Fomento al Turismo [FONATUR] (2020). Una gran visión del turismo en México. El autor. Recuperado de: https://cedocvirtual.sectur.gob.mx/janium/Documentos/000668Pri0000.pdf
García, B., Carranco, Z. y Martínez, E. (2009). El sector turístico mexicano frente a la crisis internacional. Revista El cotidiano, 24(157), 101-107.

Guzmán, M., Leyva, S. y Cárdenas, A. (2007). La relación de causalidad entre el índice bursátil mexicano y el tipo de cambio spot. Análisis Económico, 22(51), 2007, 81-105.

Instituto Nacional de Estadística Geografía e Informática [INEGI] (2019). Sistema de Cuentas Nacionales de México. Cuenta satélite del turismo de México. INEGI. Recuperado de: https://www.datatur.sectur.gob.mx/SitePages/ProductoDestacado3.aspx

Jiménez, M., Peña, A. y Ruiz, J. (2017). La demanda turística internacional: recuperación de la crisis y turismo de lujo, una primera aproximación al caso español. Journal of Regional Research, 38, 47-66.

Juárez, R. A. (2007). Análisis del Efecto de Tipo de Cambio en la Estructura Financiera de las Empresas que Cotizan en la Bolsa Mexicana de Valores; Sector Alimentos, Bebida y Tabaco. [Tesis Profesional, Facultad de Contaduría y Administración, Universidad Nacional Autónoma de México].

Kido, A., Juárez, A., Domínguez, J. y Sánchez, J. (2016). Impacto económico transversal del turismo en México. Documentos de Investigación Estadística y Económica, No. 2016-3. Secretaria de Turismo, Subsecretaria de Planeación y Política Turística. Recuperado de: https://www.datatur.sectur.gob.mx/SitePages/Iniciobanner1.aspx

Mántey, G. (2013). ¿Conviene flexibilizar el tipo de cambio para mejorar la competitividad? Revista Problemas del Desarrollo, 33(175), 9-32.

Marquina, S. (2014). Comparación y articulación interna de la actividad económica del sector turístico a partir de la Matriz de Insumo-Producto Turística de México (MIPTM-2003) basada en el enfoque de Cuenta Satélite de Turismo (CST). [Tesis doctoral, Facultad de Ciencias Económicas y empresariales, Departamento de Economía Aplicada, Universidad Autónoma de Madrid]. Recuperado de: https://repositorio.uam.es/handle/10486/662138

Medina, R. (2020). Validez de contenido de un instrumento de medición de ventaja competitiva del sector hotelero. Nova Rua, 12(21), 25-40. 
Morales, J., Velázquez, M. y García, C. (2016). La depreciación del peso mexicano durante 20122015 y su efecto en el Índice de Precios y Cotizaciones de la Bolsa Mexicana de Valores. Un análisis intersectorial. Revista Economía Informa, (397), 105-121.

Muhammand, S. M., Mohammad, A. F., Ghulam, M. S., Mubbshar, A. y Salman, M. S. (2018). Asymmetric impact of oil prices, exchange rate, and inflation on tourism demand in $\mathrm{Pa}$ kistan: new evidence from nonlinear ARDL. Asia Pacific Journal of Tourism Research, 23(4), 408-422.

Nava, F. J. (2012). El tipo de cambio como factor para promover las exportaciones. [Tesina Lic. en Relaciones Internacionales, Facultad de Ciencias Políticas y Sociales, Universidad Nacional Autónoma de México].

Organización Mundial de Turismo - OMT (2020). Barómetro OMT del Turismo Mundial. Barometer, 18(4), 1-36.

Quayson, J. y Var, T. (1982). A tourism demand function for the Okanagan, BC. Tourism Management, 3(2), 108-115.

Secretaría de Turismo [SECTUR] (2014). Compendio Estadístico del Turismo en México 2014. SECTUR, Subsecretaría de Planeación y Política Turística de México. Recuperado de: http:// www.datatur.sectur.gob.mx/Documentos\%20Publicaciones/2014_Compendio_Presentacion.pdf

Secretaría de Turismo [SECTUR] (2016). Compendio Estadístico del Turismo en México 2016. SECTUR, Subsecretaría de Planeación y Política Turística de México.
Secretaria de Turismo [SECTUR] (2019). Resultados de la actividad turística, enero de 2019. SECTUR, Subsecretaría de Planeación y Política Turística de México. Recuperado de: https://www.datatur. sectur.gob.mx/SitePages/versionesRAT.aspx

Sistema Integral sobre Economía Minera [SINEM] (2019). Históricos precios diarios petróleo WTI, Brent y MME. Recuperado de: http:// www.sgm.gob.mx/Web/SINEM/energeticos/ wti_brent_mme.html\#

Tang, C. F. y Lean, H. H. (2007). Will inflation increase crime rate? New evidence from bounds and modified Wald tests. Global Crime, 8(4), 311-323.

Torres, G. R. (2007). Teoría del comercio Internacional. Siglo veintiuno editores.

Valverde, M. y Benavides, M. (2017). El turismo en México, una visión hacia el futuro. Planur-e, Revista digital de urbanismo, (9), 1-15.

Vargas, E. (2010) El poder de los tipos de cambio. Perspectivas, 26, 9-26.

Vázquez-Martínez, G. (2019). Organización de la política y oferta de turismo en el noreste mexicano. Turismo y Sociedad, 25, 51-71.

Witt, S. F. y Martin, C. A. (1987). Econometric models for forecasting international tourism demand. Journal of Travel Research, 25(3), 2330.

Yeoman, I., Lennon, J., Blake, A., Galt, M., Greenwood, C. y McMahon-Beattie, U. (2007). Oil depletion: What does this mean for Scottish tourism? Tourism Management, 28(5), 13541365. 